\title{
A Prospective, Phase I/II, Open-Label Pilot Trial to Assess the Safety of Hyperthermic Intraperitoneal Chemotherapy After Oncological Resection of Pancreatic Adenocarcinoma
}

\author{
Can Yurttas, $\mathrm{MD}^{1}$ (D), Philipp Horvath, $\mathbf{M D}^{1}$, Imma Fischer, $\mathbf{P h D}^{2}$, Christoph Meisner, $\mathbf{P h D}^{2}$, \\ Silvio Nadalin, $\mathrm{MD}^{1}$, Ingmar Königsrainer, $\mathrm{MD}^{1,3}$, Alfred Königsrainer, $\mathrm{MD}^{1,4,5}$, Stefan Beckert, $\mathrm{MD}^{1,6}$, and \\ Markus W. Löffler, MD ${ }^{1,4,5,7,8}$
}

${ }^{1}$ Department of General, Visceral and Transplant Surgery, University Hospital Tübingen, Tübingen, Germany; ${ }^{2}$ Institute for Clinical Epidemiology and Applied Biometry, University Hospital Tübingen, Tübingen, Germany; ${ }^{3}$ Department of General, Visceral and Thoracic Surgery, Landeskrankenhaus Feldkirch, Feldkirch, Austria; ${ }^{4}$ German Cancer Consortium (DKTK) and German Cancer Research Center (DKFZ), Partner Site Tübingen, Tübingen, Germany; ${ }^{5}$ Cluster of Excellence iFIT (EXC2180) 'Image-Guided and Functionally Instructed Tumor Therapies', University of Tübingen, Tübingen, Germany; ${ }^{6}$ Department of General and Visceral Surgery, Schwarzwald-Baar Klinikum Villingen-Schwenningen, Villingen-Schwenningen, Germany; ${ }^{7}$ Interfaculty Institute for Cell Biology, Department of Immunology, University of Tübingen, Tübingen, Germany; ${ }^{8}$ Department of Clinical Pharmacology, University Hospital Tübingen, Tübingen, Germany

\begin{abstract}
Background. Pancreatic ductal adenocarcinoma (PDAC) is a common fatal disease with unfavorable prognosis, even after oncological resection. To improve survival, adding hyperthermic intraperitoneal chemotherapy (HIPEC) has been suggested. Whether HIPEC entails disproportional short-term mortality is unknown and a prospectively
\end{abstract}

\section{PREVIOUS COMMUNICATION TO A SOCIETY OR}

MEETING This work was accepted for presentation at the 27th Annual Meeting of the European Surgical Association (ESA), scheduled for May 22-23, 2020 in Cologne, Germany, but was cancelled due to the coronavirus pandemic.

Can Yurttas and Philipp Horvath have contributed equally to this work.

Supplementary Information The online version contains supplementary material available at https://doi.org/10.1245/s10434021-10187-8.

(C) The Author(s) 2021

First Received: 22 March 2021

Accepted: 1 May 2021;

Published Online: 15 June 2021

M. W. Löffler, MD

e-mail: markus.loeffler@uni-tuebingen.de determined adverse events profile is lacking. Since both pancreatic resection and HIPEC may relevantly influence morbidity and mortality, this uncontrolled single-arm, openlabel, phase I/II pilot trial was designed to assess the 30-day mortality rate, treatment feasibility, and adverse events connected with HIPEC after oncological pancreatic surgery. Methods. This trial recruited patients scheduled for PDAC resection. A sample size of 16 patients receiving study interventions was estimated to establish a predefined margin of treatment-associated short-term mortality with a power of $>80 \%$. Patients achieving complete macroscopic resection received HIPEC with gemcitabine administered at $1000 \mathrm{mg} / \mathrm{m}^{2}$ body surface area heated to $42{ }^{\circ} \mathrm{C}$ for 1 hour.

Results. Within 30 days after intervention, no patient died or experienced any adverse events higher than grade 3 that were related to HIPEC. Furthermore, treatment-related adverse events were prospectively documented and categorized as expected or unexpected. This trial supports that the actual mortality rate after PDAC resection and HIPEC is below $10 \%$. HIPEC treatment proved feasible in $89 \%$ of patients allocated to intervention. Pancreatic fistulas, as key complications after pancreas surgery, occurred in 3/13 patients under risk.

Conclusion. Combined pancreas resection and gemcitabine HIPEC proved feasible and safe, with 
acceptable morbidity and mortality. Based on these results, further clinical evaluation can be justified.

Registration Number. NCT02863471 (http://www.clinic altrials.gov).

Pancreatic ductal adenocarcinoma (PDAC) is a common fatal disease, predominantly diagnosed in advanced stage ${ }^{1}$ and therefore leaves only very limited curative treatment options. The only curative treatment for PDAC to date remains radical surgical resection, but most patients do not qualify for this treatment. ${ }^{2,3}$ Unfortunately, even after surgery, long-term outcomes remain poor and, for example, radical macroscopic resection produced 5-year survival rates of approximately $10 \%$ in a randomized controlled trial, ${ }^{4}$ which leaves a substantial unmet medical need for effective adjunct treatments in this indication. Among the suggested root causes for surgical failure are local recurrence and peritoneal dissemination. ${ }^{5}$ To eliminate remaining cancer cells, hyperthermic intraperitoneal chemotherapy (HIPEC) with gemcitabine has been suggested, although this treatment has never been prospectively evaluated. ${ }^{6,7}$

This treatment approach is supported by preclinical studies where normothermic intra-abdominal gemcitabine administration was shown to prevent peritoneal metastasis in mice. ${ }^{8}$ According to first-in-human pharmacokinetic studies, intraperitoneal administration of gemcitabine is well-tolerated, with provisional data suggesting low toxicity and a manageable adverse event profile, even when left in situ for 24 hours. ${ }^{9,10}$ Preliminary evidence from 21 patients after PDAC resection suggested that gemcitabine HIPEC might prove effective to prevent peritoneal metastasis. ${ }^{11}$ In this study however, associated morbidity and mortality rates (at 9.5\%) were substantial. In oncological pancreas surgery, acute mortality is also common ${ }^{12}$ and high 30-day mortality rates have been reported, ${ }^{13}$ as well as considerable morbidity rates (of up to $60 \%$ ). ${ }^{14}$

HIPEC treatment, which is usually combined with cytoreductive surgery (CRS), may also involve increased morbidity and mortality, e.g. in peritoneal metastasis from colorectal cancer where morbidity rates between 23 and $45 \%$ were reported, along with acute mortality ranging from 0 to $12 \% .^{15}$

In spite of the high unmet medical need and preliminary preclinical and clinical evidence encouraging pre-emptive HIPEC for PDAC, ${ }^{7}$ as well as premature reports of efficacy, ${ }^{11}$ robust data supporting any wider clinical application remain absent. The PanHIPEC trial was therefore designed as a non-randomized, single-arm pilot trial aiming to prospectively investigate 30-day mortality and to establish an adverse events profile, as well as the feasibility of HIPEC in patients after complete oncological PDAC resection to manage the risk of implementing this treatment for possible further clinical development.

\section{METHODS}

\section{Trial Design}

The PanHIPEC trial was prospectively designed as an uncontrolled, open label, single-arm pilot study, enrolling patients with imaging signs of non-metastatic PDAC scheduled for elective surgery. The primary study endpoint was to assess the 30-day mortality of participants after oncological surgery and HIPEC; a 30-day mortality rate of $10 \%$ was defined as a benchmark based on contemporary literature ${ }^{12,13,15}$ and considerations of risk-benefit. Secondary endpoints included assessment of safety and feasibility of treatment. Patients were interviewed and examined for severe adverse events, categorized according to the National Cancer Institute Common Terminology Criteria for Adverse Events (NCI CTCAE) version 4.0. As a trial-stopping rule, the occurrence of more than one death within 30 days after HIPEC was set. Since this trial was not designed to assess any oncological outcomes, respective results and survival data should be considered as exploratory and are only provided as a supplement.

\section{Trial Approval and Consent to Participate}

The clinical trial was conducted in accordance with the principles of the Declaration of Helsinki as well as all applicable laws and regulations, and approval was obtained from the local Institutional Review Board (Project No. 426/2015AMG1) and the Federal German Institute for Drugs and Medical Devices (BfArM). The trial was registered with ClinicalTrials.gov (identifier: NCT02863471) and the European Union Clinical Trials Register (EudraCT Number 2015-002288-41) and conforms to International Committee of Medical Journal Editors (ICMJE) and Consolidated Standards of Reporting Trials (CONSORT) guidelines, where applicable. All patients $(n=20)$ gave appropriate informed consent, documented in writing, with full understanding of the experimental procedures prior to study enrollment and interventions.

\section{Inclusion and Exclusion Criteria}

Patients aged $\geq 18$ years with a Karnofsky performance status $\geq 70 \%$ ( $=$ Eastern Cooperative Oncology Group [ECOG] status $\leq 1^{16}$ ), with suspected PDAC according to imaging findings, but without evidence for distant 
metastasis and assessed as oncologically resectable, were allocated to treatment.

Exclusion criteria included participation in competing interventional trials, secondary malignant disease within 5 years before enrollment (except for basal cell carcinoma and curatively treated cervix carcinoma in situ), prior cytostatic therapy, or contraindications/hypersensitivity to gemcitabine. Patients deemed inoperable due to severe secondary illness, e.g. severe heart failure, coronary artery disease, arrhythmias or hypertension, and pulmonary or renal impairments, were excluded. Contraception standards for interventional clinical trials applied to this study, excluding pregnant or lactating women as well as men or women of childbearing potential not consenting to preemptive use of contraception measures. Before informed consent and study inclusion, a full medical history was taken, complete physical examination and laboratory testing were performed, and radiological scans evaluated. For women of childbearing potential, negative pregnancy tests were mandatory.

\section{Surgical Treatment and Hyperthermic Intraperitoneal Chemotherapy}

After laparotomy, the abdomen was inspected for metastasis to the liver, non-locoregional lymph nodes, or the peritoneum, as well as other factors precluding full oncological resection. A tumor biopsy and intrasurgical frozen section analysis were required to confirm the diagnosis. Subsequent resection and reconstruction was performed according to disease location and extent, and closed HIPEC ${ }^{17}$ with gemcitabine $1000 \mathrm{mg} / \mathrm{m}^{2}$ body surface area ${ }^{18}$ was administered. Gemcitabine is an approved drug but is not labeled for the described use. The sutured abdomen was filled with up to $5.0 \mathrm{~L}$ of $0.9 \%$ saline, heated to $42{ }^{\circ} \mathrm{C}$, and drugs added and circulated for $60 \mathrm{~min}$ (Performer HT, RanD Biotech, Medolla, Italy). Afterwards, the abdomen was flushed with $6.0 \mathrm{~L}$ of saline and reopened for definite wound closure after exploration.

\section{Evaluated Patient Cohort and Follow-Up}

Enrolled patients allocated to HIPEC comprise the intention-to-treat (ITT) group, whereas the modified ITT (mITT) group encompasses only patients with an intraoperatively confirmed PDAC diagnosis, where an oncological resection was achieved and study treatment was applied. Only mITT patients were followed for survival and secondary endpoints. Prospective patient assessment included patient history and daily physical examinations and interviews, enquiring for predefined expected adverse events connected with gemcitabine. During hospitalization, daily laboratory tests encompassed automated differential blood counts, electrolytes, retention values, hepatic transaminases, cholestasis parameters, $\alpha$-amylase, and lipase; $\alpha$ amylase and lipase were also determined on a daily basis from abdominally inserted drains. At discharge before day 30 postsurgery, follow-up was continued by telephone; after day 30 postsurgery, a concluding end-of-trial visit with interviews and laboratory and physical examinations was performed.

\section{Statistics and Sample Size Determination}

Thirty-day mortality was prospectively defined as the primary endpoint for statistical evaluation. A one-sided exact $95 \%$ confidence interval (CI) and a one-sided exact binomial test were used to evaluate the null hypothesis that the observed 30-day mortality rate is $\geq 10 \%$, against the alternative that the 30-day mortality rate is $<10 \%$. With a required number of 16 patients, the probability (power in terms of the exact binomial probability) of experiencing a critical incident (death within 30 days after surgery) for at least one patient is $81.5 \%$, if the incidence of critical events is $\geq 10 \%$ in the main population. For statistical analysis, the exact one-sided binomial test was used.

\section{Assessment of Endpoints}

As the primary endpoint, the incidence of death within the mITT population (with 95\% CI) was analyzed. Additionally, the probability of 30-day survival was compared with the minimal acceptable probability of $\pi_{0}=0.9$ using the one-sided binomial test.

Secondary endpoints included adverse events and feasibility of treatment. Feasibility was assessed as the frequency of patients receiving HIPEC among the ITT group. Patients were interviewed and examined for adverse events occurring within 30 days after HIPEC, which were categorized as expected adverse events or unexpected adverse events and graded according to CTCAE version 4.0. Gemcitabine-related expected adverse events were predefined and deduced from intravenous gemcitabine administration ${ }^{19}$ as neutropenia, thrombocytopenia, nausea, vomitus, diarrhea, stomatitis, and hair loss. Surgical complications ascribable to pancreatic surgery were specified in the study protocol and omitted among adverse events. Pancreatic fistulas as key surgical complications were documented and graded according to the classification of postoperative pancreatic fistulas (POPF) of the International Study Group of Pancreatic Surgery (ISGPS). ${ }^{20}$

\section{Exploratory Analyses}

Overall survival (OS) of patients was assessed using Kaplan-Meier and Cox regression analysis, as well as log- 
rank analyses, and plotted using SPSS version 24 (IBM Corporation, Armonk, NY, USA). Further graphs were plotted using GraphPad Prism version 8 (GraphPad Software, La Jolla, CA, USA). Long-term follow-up for exploratory survival analyses was conducted by telephone. Due to the exploratory nature of these analyses, the respective information is provided as a supplement.

\section{RESULTS}

\section{Patient Characteristics, Screening, and Study Enrollment}

Between December 2015 and July 2017, 20 patients [13 men and 7 women, median age 62.5 years (range 50-79 years); median body surface area $1.8 \mathrm{~m}^{2}$ (range 1.5-2.3)] were assessed for eligibility. Of 20 screened patients scheduled for surgery, two patients were excluded due to extended disease. Among the remaining patients $(n=18$; ITT), two patients were excluded from HIPEC during surgery-one patient due to histologically unconfirmed PDAC diagnosis and the other for severe hemorrhagic diathesis with edema of the gut. Ultimately, a total of 16 patients with confirmed PDAC diagnosis received HIPEC after oncological resection (mITT; $n=16$ ) (Fig. 1). Patient characteristics, tumor staging and treatment details are provided in Table 1.

\section{Description of the Intention-to-Treat (ITT) and Modified ITT (mITT) Populations}

Surgical procedures in the ITT group $(n=18)$ included partial pancreatoduodenectomy in 11 patients, distal pancreatectomy and splenectomy in 4 patients, and total pancreatectomy and splenectomy in 3 patients. Overall, 16/18 patients (mITT group) received HIPEC (details are provided in Table 2). In all patients in the mITT group, the PDAC diagnosis initially made from intrasurgical frozen sections was affirmed by the subsequent definite histological examination. Microscopic disease (R1) remained in 10 of these patients, whereas the resection margins were assessed as tumor-free (R0) in only $6 / 16$ patients.

\section{Mortality, Safety, and Feasibility}

In the mITT group, no postoperative mortality was observed within the first 30 days $(95 \%$ CI $0-0.172$; $p=0.091$ ), and the primary endpoint of the trial was therefore reached. None of the patients experienced any documented adverse events higher than grade 3 (Fig. 2a). Overall, 62 adverse events were documented, of which half $(n=31)$ were categorized as expected adverse events and the other half were categorized as unexpected adverse events (see Table 3). Most adverse events were categorized as mild (grade $1 ; 36 / 62$ ), about one-third were categorized as moderate (grade $2 ; 20 / 62$ ), and others were categorized as severe (grade $3 ; 6 / 62$ ). The maximum documented severity of expected adverse events was grade 3 (severe), affecting one patient. In addition to these adverse events predefined as 'expected', a total of 31 unexpected adverse events affected 15 patients. The maximum extent of unexpected adverse events was also severe (grade 3), affecting four patients in whom five severe adverse events overall were documented. A relation of unexpected adverse events to the study intervention with HIPEC was assessed as probable or possible in two patients, whereas this was evaluated as unlikely in 12 occasions of unexpected adverse events. About half of the unexpected adverse events were considered not related to HIPEC treatment. Single patients were affected by up to three expected adverse events and five unexpected adverse events in the course of postoperative surveillance (Fig. 2b). When describing surgical complications according to the Clavien-Dindo Classification ${ }^{21}$, the maximum grade was IVb, affecting one patient with leakage of the pancreatic anastomosis with a septic shock requiring prolonged intensive care. Grade IIIa interventions for surgical site infection and myocardial infarction were necessary in three patients. Overall, the majority of adverse events were considered as the minimal deviation from postoperative course (i.e. grade I) or requiring pharmacologic treatment (grade II) (see Table 3). Feasibility was assessed as the percentage of patients with histologically confirmed PDAC receiving HIPEC after oncological resection $(n=16)$ among all patients allocated to intervention $(n=18)$. This fraction included $89 \%$ of patients.

\section{Pancreatic Fistulas}

Pancreatic fistulas occurred in 3/13 patients under risk, omitting 3 patients in the mITT group who received a total pancreatectomy. Two patients were treated conservatively (both grade A according to the POPF classification), whereas one patient underwent a resection of the pancreatic anastomosis due to anastomotic leakage with consecutive septic shock and temporary organ failure, receiving external drainage of the pancreatic duct (grade C). Hereupon the patient slowly recovered and was discharged home 24 days after surgery. The patient experienced rapid progressive disease 2 months later and passed away after 21 months due to malignant obstruction ileus. All patients were treated according to implemented standards without any alterations due to HIPEC therapy. 
TABLE 1. Patient characteristics, tumor staging, and treatment

\begin{tabular}{|c|c|}
\hline & $\begin{array}{l}\text { Surgery with HIPEC (mITT) } \\
{[n=16]}\end{array}$ \\
\hline \multicolumn{2}{|l|}{ Ethnicity } \\
\hline Caucasian & 16 \\
\hline \multicolumn{2}{|l|}{ Sex } \\
\hline Female & 5 \\
\hline Male & 11 \\
\hline Median age, years (IQR) & $62.5(55.0-67.5)$ \\
\hline \multicolumn{2}{|l|}{ Karnofsky Index } \\
\hline $100 \%$ & 13 \\
\hline $90 \%$ & 2 \\
\hline $80 \%$ & 1 \\
\hline \multicolumn{2}{|l|}{ Location of tumor } \\
\hline Head & 10 \\
\hline Body & 2 \\
\hline Tail & 2 \\
\hline Unassignable & 2 \\
\hline \multicolumn{2}{|l|}{ Tumor extension } \\
\hline $\mathrm{T} 1$ & 0 \\
\hline $\mathrm{T} 2$ & 7 \\
\hline $\mathrm{T} 3$ & 9 \\
\hline $\mathrm{T} 4$ & 0 \\
\hline \multicolumn{2}{|l|}{ Nodal status } \\
\hline No & 3 \\
\hline N1 & 10 \\
\hline $\mathrm{N} 2$ & 3 \\
\hline \multicolumn{2}{|l|}{ Metastasis } \\
\hline M0 & 16 \\
\hline M1 & 0 \\
\hline \multicolumn{2}{|l|}{ Stage } \\
\hline IA & 0 \\
\hline IB & 3 \\
\hline IIA & 0 \\
\hline IIB & 9 \\
\hline III & 4 \\
\hline IV & 0 \\
\hline \multicolumn{2}{|l|}{ Grade of differentiation } \\
\hline G1 & 0 \\
\hline G2 & 7 \\
\hline G3 & 7 \\
\hline G4 & 2 \\
\hline \multicolumn{2}{|l|}{ Type of resection } \\
\hline PPD & 10 \\
\hline DP & 3 \\
\hline $\mathrm{TP}$ & 3 \\
\hline \multicolumn{2}{|l|}{ Resection status } \\
\hline R0 & 6 \\
\hline $\mathrm{R} 1$ & 10 \\
\hline $\begin{array}{l}\text { Median duration of surgery, } \\
\min (\mathrm{IQR})\end{array}$ & $426(339-498)$ \\
\hline
\end{tabular}

TABLE 1. continued

\begin{tabular}{l}
\hline \multicolumn{1}{c}{$\begin{array}{l}\text { Surgery with HIPEC (mITT) } \\
{[n=16]}\end{array}$} \\
$\begin{array}{l}\text { Median duration of hospital stay, } \\
\text { days (IQR) }\end{array}$ \\
\hline $\begin{array}{l}\text { Data are expressed as } n \text { unless otherwise specified and are tabulated } \\
\text { for the mITT group }\end{array}$ \\
Resection status was determined during postoperative histopatho- \\
logical work-up \\
TNM classification and staging is provided according to the UICC \\
classification of malignant tumors, 8th edition \\
$D P$ distal pancreatectomy, HIPEC hyperthermic intraperitoneal \\
chemotherapy, $I Q R$ interquartile range, $m I T T$ modified intention-to- \\
treat group, $P P D$ partial pancreatoduodenectomy, $T P$ total pancrea- \\
tectomy, $U I C C$ Union for International Cancer Control
\end{tabular}

\section{Post-Interventional Follow-Up and Mortality}

After trial termination, patients included in the mITT group were further followed to determine OS after PDAC surgery and HIPEC for exploratory analysis. The median OS of this cohort was 16.1 months after surgery and the 1-year survival rate was $62.5 \%(n=10)$ (electronic supplementary Fig. 1). Overall, no short-term mortality was evidenced, neither after 30 days nor 90 days after CRS and HIPEC. The first patient died 4.4 months after HIPEC due to tumor progression of an anaplastic PDAC. At the end of follow-up (approximately 24 months), five patients remained alive or of unknown status (electronic supplementary Table 1).

\section{DISCUSSION}

In spite of a theoretical rationale for performing HIPEC after PDAC resection, ${ }^{6,7}$ robust safety data are missing and potential downsides remain unknown. HIPEC was not only proposed as an adjunct treatment ${ }^{6,7}$ but has also already been administered to PDAC patients, both after oncological resection ${ }^{7,11,22}$ and following CRS for peritoneal metastasis. ${ }^{23}$ Although HIPEC has been shown to be comparably safe among high-risk surgical oncology procedures,${ }^{24}$ evidence is lacking that a combination of pancreatic surgery and HIPEC does not cause disproportional cumulative mortality and major morbidity, surpassing its potential benefits. In many clinical indications where HIPEC is discussed, pilot studies formally assessing mortality and prospective adverse event profiles are lacking regardless of their broad clinical application. ${ }^{25}$ Recent work comparable with our study, performed in peritoneal metastasis from gastric cancer, has shown substantial treatment-related mortality, with $50 \%$ mortality at the highest investigated 
FIG. 1. Enrollment, allocation, follow-up, and analysis of patients during the trial (the layout was adapted from the CONSORT 2010 statement). ${ }^{43}$ ITT intention-to-treat group, mITT modified intention-to-treat group, PDAC pancreatic ductal adenocarcinoma

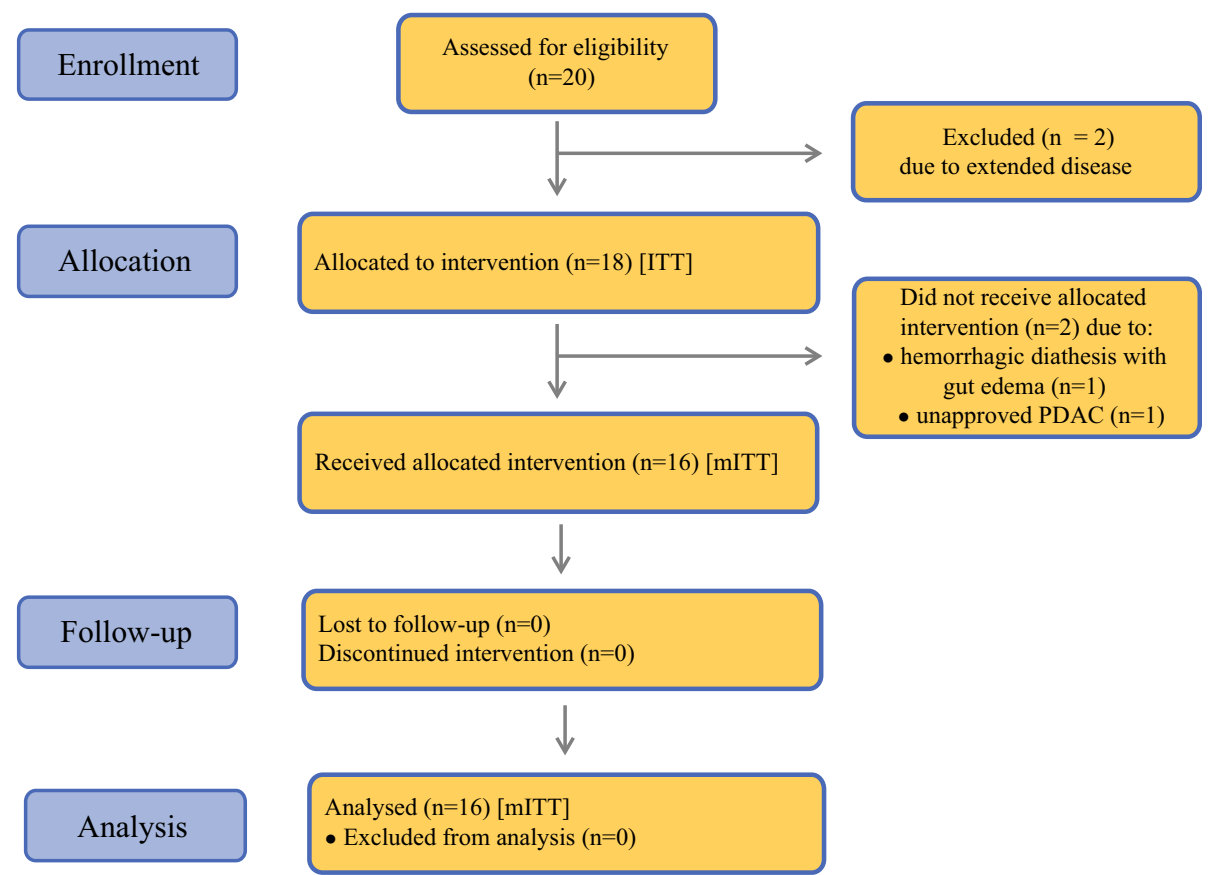

TABLE 2. HIPEC

characteristics

\begin{tabular}{ll}
\hline Parameters & \\
\hline Total administered drug dose, mg [median (IQR)] & $1890(1800-1930)$ \\
Volume of carrier solution, L & 5.0 \\
Drug concentration, mg/L & $378(360-386)$ \\
Temperature, ${ }^{\circ} \mathrm{C}[$ median $(\mathrm{IQR})]$ & $41.5(41.5-41.9)$ \\
Flow rate, $\mathrm{mL} / \mathrm{min}[$ median $(\mathrm{IQR})]$ & $1250(1200-1285)$ \\
Duration, $\mathrm{min}$ & 60
\end{tabular}

HIPEC treatment was performed with gemcitabine administered at $1000 \mathrm{mg} / \mathrm{m}^{2}$ BSA in the modified intention-to-treat group $(n=16)$

HIPEC hyperthermic intraperitoneal chemotherapy, BSA body surface area, IQR interquartile range dose level of docetaxel in combination with oxaliplatin. ${ }^{26}$ Furthermore, serious adverse events were reported in 17/25 patients.

It is therefore against this background that we aimed to assess treatment-associated short-term mortality in a pilot trial, for combining both procedures. Interestingly, even in peritoneal metastasis, where pancreatic resection was considered a contraindication for CRS and HIPEC due to a high complication risk, recent data suggest it may be defensible. $^{27}$ Based on risk-benefit considerations and mortality rates from the literature for both procedures, ${ }^{12,13,15}$ a benchmark was set at 10\% 30-day mortality, assuming that for the investigated clinical setting, mortality rates $\geq 10 \%$ could question further clinical development. Our study now suggests that HIPEC does not substantially contribute to acute mortality since the mortality of the combined procedure was determined below $10 \%$ due to the absence of deaths during the observation period (30 days after PDAC surgery and HIPEC; primary endpoint). Therefore, we conclude that adding gemcitabine HIPEC to pancreatic resection can be considered sufficiently safe in experienced medical centers.

Previously consolidated adverse event profiles for HIPEC with gemcitabine were unavailable and hitherto only anecdotal data were accessible, e.g. Sugarbaker et al. merely reported no grade 3 or 4 toxicities $^{7}$ and Tentes et al. refer to one case of afebrile neutropenia (grade 2). ${ }^{11} \mathrm{We}$ have witnessed expected adverse events and previously undescribed adverse events in all but one patient in the immediate postoperative course with a maximum of grade 3. Such severe adverse events were reported in six instances, rendering the adverse event profile acceptable in our view. Pancreatic fistulas are reported as frequent complications after pancreas surgery, particularly when 


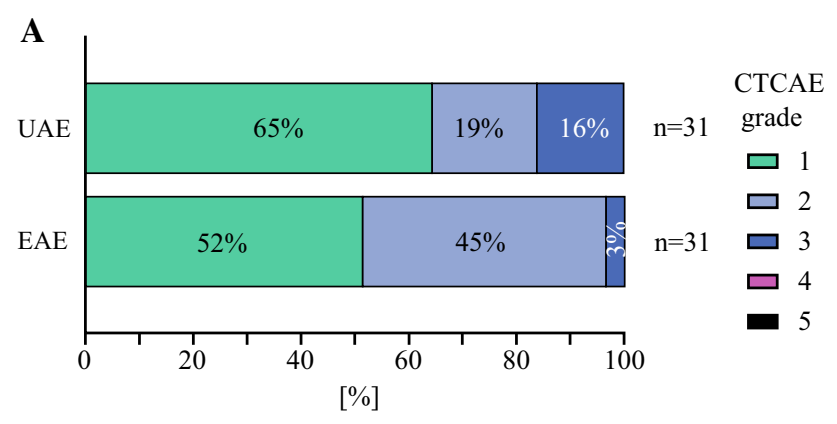

B
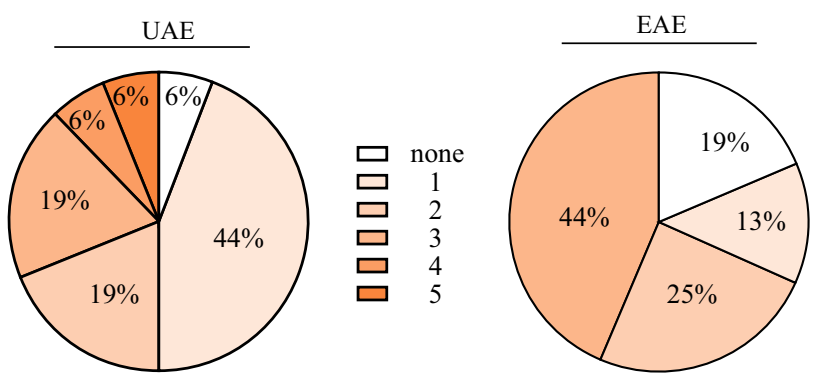

FIG. 2. Adverse event (AE) overview in the mITT group. a Proportional distribution (\%) of AEs classified according to National Cancer Institute CTCAE v.4.0, categorized as EAEs (bottom column) or UAEs (top column) [please see the Methods section (Assessment of Endpoints) for respective details]. b Proportional distribution (\%) of patients experiencing (n) cumulative UAEs (left)/EAEs (right) over 30 days postinterventional follow-up. mITT modified intention-to-treat group, UAEs unexpected adverse events, EAEs expected adverse events, CTCAE Common Terminology Criteria for Adverse Events, AE adverse event

combined CRS and HIPEC is performed, ${ }^{28}$ frequently involving severe complications and potentially increasing mortality. ${ }^{27}$ Such complications occurred in three patients in our study cohort (two grade $\mathrm{A}$ and one grade $\mathrm{C}$ according to the POPF classification ${ }^{20}$ ), among 13 patients under risk. These results are comparable with respective complication rates reported in the literature after pancreas surgery ${ }^{29}$ however, since 3 of the 16 patients in the mITT cohort received a total pancreatectomy, this may have reduced the overall risk profile and special attention should be paid to the treatment-associated risk for POPF in any future trials. On follow-up after trial termination, there was no 90-day mortality and the first HIPEC patient died 4.4 months after surgery.

Due to the early stage of development and the small cohort size, this prospective clinical trial comes with heavy limitations, particularly regarding any ancillary clinical or oncological endpoints, since this trial was not designed to reliably assess such endpoints (including OS, comparisons with established treatments, etc.). Therefore, the presented data have to be considered as exploratory and without (immediate) relevance for patient care. Of note, whether
HIPEC proves effective in PDAC by adding any benefits, such as increased long-term survival or disease control, remains to be answered. Further prevailing issues are, for instance, the remaining microscopic disease in most patients $(n=10)$ and that current adjuvant treatment options have not been used, as well as the highly variable mortality and morbidity observed among German centers ${ }^{30}$ that are influenced by patient- and treatment-intrinsic variables, a fact that ultimately precludes any valid assessment of oncological benefits in this case.

An immanent design aspect of this clinical trial is that no control group without HIPEC treatment has been included, which does limit the precise attribution of adverse events to specific parts of the investigated treatment. Furthermore, the heterogeneous patient cohort and surgical treatment may raise legitimate critique in the context of oncological benefits, whereas these circumstances do not interfere with the primary endpoints of this trial (see also van der Kaaij et al. ${ }^{26}$ ).

On the other hand, there are clear and defined benefits of this prospective clinical trial that include robust safety data, which hitherto were unavailable. It should also be appreciated that this is the first ever prospectively planned and conducted HIPEC trial in this indication. Considering unsystematic previous clinical tests of the approach (as described e.g. by Tentes et al. ${ }^{22}$ ), our robust trial design and predefined aims add validity and can therefore answer relevant clinical questions in a more definitive manner.

Because, for example, in colorectal cancer where HIPEC was evaluated after $\mathrm{CRS}^{31}$ as well as for the prevention of peritoneal metastasis ${ }^{32}$ the latest randomized controlled trials did not confirm a clinical benefit, an ongoing controversy has evolved regarding the role of the HIPEC treatment. ${ }^{33-37} \mathrm{~A}$ recent systematic review on intraperitoneal chemotherapy in PDAC patients could only identify data on 85 patients in eight publications. Based on these limited data, the treatment was concluded to be well tolerated and potentially suited for short-term disease control, whereas adjuvant HIPEC in resectable PDAC was discouraged. ${ }^{38}$ Although significant oncological effectiveness of HIPEC in PDAC may emerge as doubtful, such comprehensive conclusions based on very limited and preliminary evidence may likewise be questioned. Since HIPEC is influenced by a multitude of factors, ${ }^{39,40}$ preclinical research and modeling appears pivotal as a guide for the future..$^{37,41,42}$ Nevertheless, we are convinced that this pilot trial has added relevant information, providing solid knowledge regarding adverse events to be expected with gemcitabine HIPEC, and fundamental evidence on mortality risks incurred when adding HIPEC after PDAC resection, essential facts that are required before any possible further clinical development should be considered. 
TABLE 3. Treatment-emergent expected adverse events (EAEs) and unexpected adverse events (UAEs)

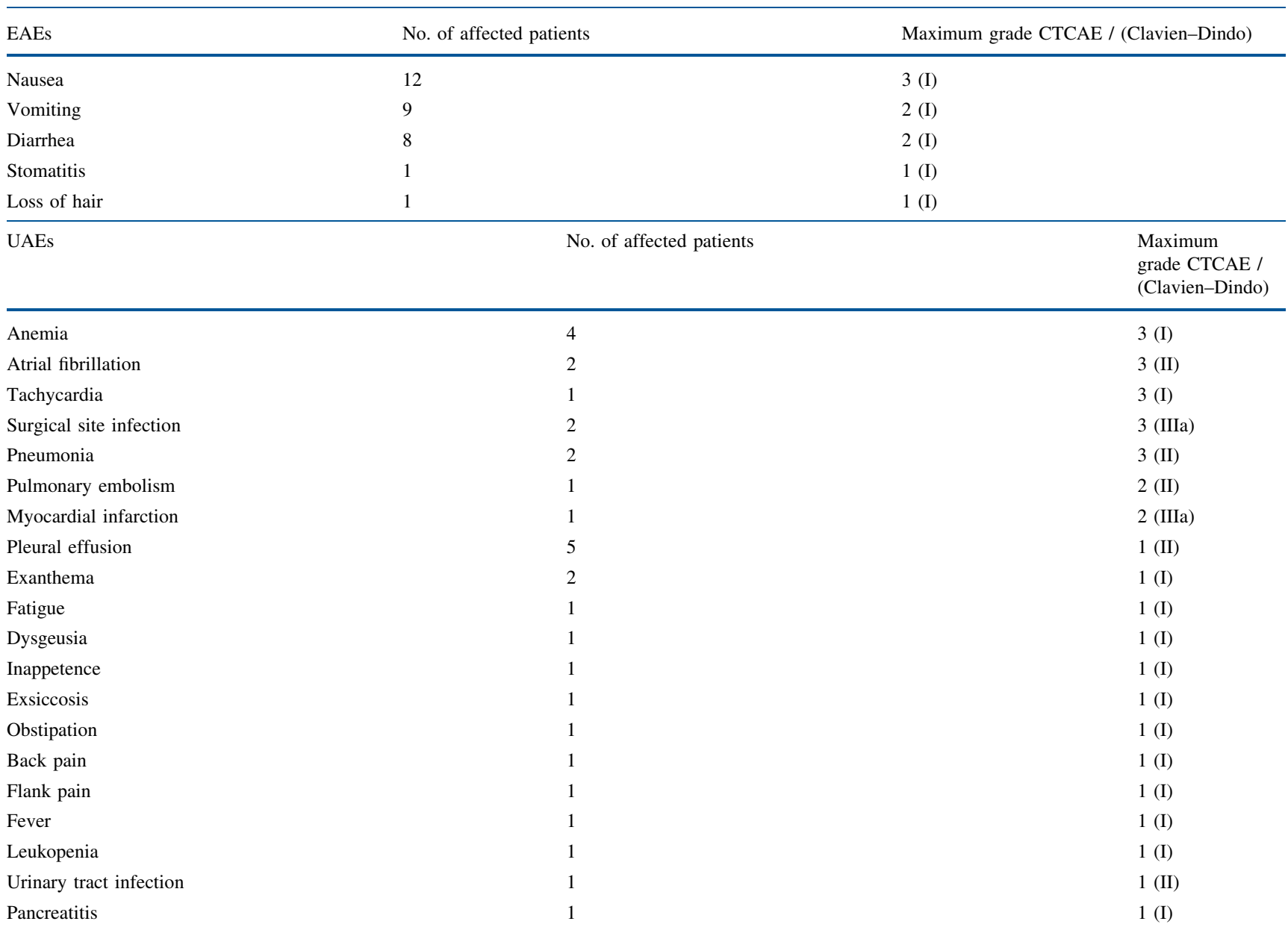

AEs documented in the modified intention-to-treat group $(n=16)$ are classified as EAEs $(n=31)$ or UAEs $(n=31)$ and are organized according to maximum severity (CTCAE v.4.0) and number of patients affected, including corresponding grades according to the Clavien-Dindo classification (not foreseen in the trial protocol)

EAEs expected adverse events, UAEs unexpected adverse events, CTCAE Common Terminology Criteria for Adverse Events, AEs adverse events

ACKNOWLEDGMENT The authors would like to dedicate this article to the memory of Prof. Dr. Sebastian P. Haen, University Medical Center Hamburg-Eppendorf, and acknowledge his contributions to this trial and the preparation of this manuscript. Prof. Haen died sadly before this manuscript was accepted for publication. Furthermore, the authors wish to thank Silvia Wagner, $\mathrm{PhD}$, and Silke Templin, MD, of the study center at the Department of General, Visceral and Transplant Surgery, and their staff, Jürgen Winter, Archana Yoganandarajah and Sonja Heselmaier-Troll, for excellent support with regard to patient recruitment, trial conduct, and data collection. Finally, the authors are sincerely thankful to all participating patients and their families. This research was registered on August 11, 2016 at http://www.clinicaltrials.gov, under registration number NCT02863471, stating the planned key analyses for this prospective clinical trial.
AUTHOR CONTRIBUTIONS SB, CM and MWL conceived the study and designed the clinical trial. SB and MWL obtained funding. $\mathrm{CM}$ and IF performed sample size determination, data curation, and statistical analysis, and provided support. $\mathrm{CY}, \mathrm{PH}, \mathrm{IK}, \mathrm{AK}, \mathrm{SB}$ and MWL managed and conducted the clinical trial. CY, PH, IF, CM, SN, IK, AK, SB and MWL performed patient enrollment, treatment, and data collection, as well as analysis and interpretation of the data. CY, $\mathrm{PH}$ and MWL conceived the manuscript. CY and MWL wrote the first draft of the manuscript. PH, IF, CM, SN, AK and SB critically revised the manuscript for important intellectual content. PH and SB have overall responsibility for the trial conduct, and MWL is the overall guarantor for the study and the manuscript. All authors approved the final version of the manuscript.

FUNDING Open Access funding enabled and organized by Projekt DEAL. This work was supported by a grant from the Medical Faculty at the University of Tübingen, Germany; the 'Angewandte Klinische 
Forschung' (AKF) program for investigator-initiated clinical trials (Project No. 338-0-0). The funder was not involved in the study design, data collection, data analysis, manuscript preparation and publication decisions.

DISCLOSURE Markus W. Löffler and Alfred Königsrainer received a research grant from RanD S.r.l., the manufacturer of devices and consumables for HIPEC, unrelated to the present work. Can Yurttas, Philipp Horvath, Imma Fischer, Christoph Meisner, Silvio Nadalin, Ingmar Königsrainer, and Stefan Beckert declare no potential conflicts of interest.

OPEN ACCESS This article is licensed under a Creative Commons Attribution 4.0 International License, which permits use, sharing, adaptation, distribution and reproduction in any medium or format, as long as you give appropriate credit to the original author(s) and the source, provide a link to the Creative Commons licence, and indicate if changes were made. The images or other third party material in this article are included in the article's Creative Commons licence, unless indicated otherwise in a credit line to the material. If material is not included in the article's Creative Commons licence and your intended use is not permitted by statutory regulation or exceeds the permitted use, you will need to obtain permission directly from the copyright holder. To view a copy of this licence, visit http://creativecommons. org/licenses/by/4.0/.

\section{REFERENCES}

1. Azar I, Virk G, Esfandiarifard S, Wazir A, Mehdi S. Treatment and survival rates of stage IV pancreatic cancer at VA hospitals: a nation-wide study. J Gastrointest Oncol. 2019;10(4):703-11.

2. Ansari D, Gustafsson A, Andersson R. Update on the management of pancreatic cancer: surgery is not enough. World $J$ Gastroenterol. 2015;21(11):3157-65.

3. Geer RJ, Brennan MF. Prognostic indicators for survival after resection of pancreatic adenocarcinoma. Am $J$ Surg. 1993;165(1):68-73.

4. Oettle H, Neuhaus P, Hochhaus A, et al. Adjuvant chemotherapy with gemcitabine and long-term outcomes among patients with resected pancreatic cancer: the CONKO-001 randomized trial. JAMA. 2013;310(14):1473-81.

5. Griffin JF, Smalley SR, Jewell W, et al. Patterns of failure after curative resection of pancreatic carcinoma. Cancer. 1990;66(1):56-61.

6. Kamath A, Yoo D, Stuart OA, Bijelic L, Sugarbaker PH. Rationale for an intraperitoneal gemcitabine chemotherapy treatment for patients with resected pancreatic cancer. Recent Pat Anticancer Drug Discov. 2009;4(2):174-9.

7. Sugarbaker PH, Stuart OA, Bijelic L. Intraperitoneal gemcitabine chemotherapy as an adjuvant treatment for patients with resected pancreatic cancer: phase II and pharmacologic studies. Transl Gastrointest Cancer. 2012;1(2):161-8.

8. Ridwelski K, Meyer F, Hribaschek A, Kasper U, Lippert H. Intraoperative and early postoperative chemotherapy into the abdominal cavity using gemcitabine may prevent postoperative occurence of peritoneal carcinomatosis. J Surg Oncol. 2002;79(1):10-6.

9. Gamblin TC, Egorin MJ, Zuhowski EG, et al. Intraperitoneal gemcitabine pharmacokinetics: a pilot and pharmacokinetic study in patients with advanced adenocarcinoma of the pancreas. Cancer Chemother Pharmacol. 2008;62(4):647-53.

10. Sugarbaker PH, Stuart OA, Bijelic L. Intraperitoneal gemcitabine chemotherapy treatment for patients with resected pancreatic cancer: rationale and report of early data. Int J Surg Oncol. 2011;2011:161862.

11. Tentes AA, Kyziridis D, Kakolyris S, et al. Preliminary results of hyperthermic intraperitoneal intraoperative chemotherapy as an adjuvant in resectable pancreatic cancer. Gastroenterol Res Pract. 2012;2012:506571.

12. Halloran CM, Ghaneh P, Bosonnet L, Hartley MN, Sutton R, Neoptolemos JP. Complications of pancreatic cancer resection. Dig Surg. 2002;19(2):138-46.

13. Bakkevold KE, Kambestad B. Morbidity and mortality after radical and palliative pancreatic cancer surgery. Risk factors influencing the short-term results. Ann Surg. 1993;217(4):356-68.

14. Gouma DJ, van Geenen RC, van Gulik TM, et al. Rates of complications and death after pancreaticoduodenectomy: risk factors and the impact of hospital volume. Ann Surg. 2000;232(6):786-95.

15. Glockzin G, Ghali N, Lang SA, Schlitt HJ, Piso P. Results of cytoreductive surgery and hyperthermic intraperitoneal chemotherapy for peritoneal carcinomatosis from colorectal cancer. J Surg Oncol. 2009;100(4):306-10.

16. Verger E, Salamero M, Conill C. Can Karnofsky performance status be transformed to the eastern cooperative oncology group scoring scale and vice versa? Eur $J$ Cancer. 1992;28A(8-9):1328-30.

17. Esquivel J, Elias D, Baratti D, Kusamura S, Deraco M. Consensus statement on the loco regional treatment of colorectal cancer with peritoneal dissemination. J Surg Oncol. 2008;98(4):263-7.

18. Du Bois D, Du Bois EF. A formula to estimate the approximate surface area if height and weight be known. Arch Intern Med. 1916;17:863-71.

19. Toschi L, Finocchiaro G, Bartolini S, Gioia V, Cappuzzo F. Role of gemcitabine in cancer therapy. Future Oncol. 2005;1(1):7-17.

20. Bassi C, Marchegiani G, Dervenis C, et al. The 2016 update of the International Study Group (ISGPS) definition and grading of postoperative pancreatic fistula: 11 years after. Surgery. 2017;161(3):584-91.

21. Dindo D, Demartines N, Clavien PA. Classification of surgical complications: a new proposal with evaluation in a cohort of 6336 patients and results of a survey. Ann Surg. 2004;240(2):205-13.

22. Tentes AA, Stamou K, Pallas N, Karamveri C, Kyziridis D, Hristakis C. The effect of hyperthermic intraoperative intraperitoneal chemotherapy (HIPEC) as an adjuvant in patients with resectable pancreatic cancer. Int J Hyperth. 2016;32(8):895-9.

23. Tentes AA, Pallas N, Karamveri C, Kyziridis D, Hristakis C. Cytoreduction and HIPEC for peritoneal carcinomatosis of pancreatic cancer. J BUON. 2018;23(2):482-7.

24. Foster JM, Sleightholm R, Patel A, et al. Morbidity and chemotherapy compared with other high-risk surgical oncology procedures. JAMA Netw Open. 2019;2(1):e186847.

25. Gill RS, Al-Adra DP, Nagendran J, et al. Treatment of gastric cancer with peritoneal carcinomatosis by cytoreductive surgery and HIPEC: a systematic review of survival, mortality, and morbidity. J Surg Oncol. 2011;104(6):692-8.

26. van der Kaaij RT, Wassenaar ECE, Koemans WJ, et al. Treatment of peritoneal disease in stomach cancer with cytoreductive surgery and hyperthermic intraperitoneal chemotherapy: PERISCOPE I initial results. Br J Surg. 2020;107(11):1520-8.

27. Schwarz L, Votanopoulos K, Morris D, et al. Is the combination of distal pancreatectomy and cytoreductive surgery with HIPEC reasonable? Results of an international multicenter study. Ann Surg. 2016;263(2):369-75.

28. Downs-Canner S, Ding Y, Magge DR, et al. A comparative analysis of postoperative pancreatic fistulas after surgery with and 
without hyperthermic intraperitoneal chemoperfusion. Ann Surg Oncol. 2015;22(5):1651-7.

29. McMillan MT, Soi S, Asbun HJ, et al. Risk-adjusted outcomes of clinically relevant pancreatic fistula following pancreatoduodenectomy: a model for performance evaluation. Ann Surg. 2016;264(2):344-52.

30. Krautz C, Denz A, Weber GF, Grutzmann R. Influence of hospital volume effects and minimum caseload requirements on quality of care in pancreatic surgery in Germany. Visc Med. 2017;33(2):131-4.

31. Quenet F, Elias D, Roca L, et al. Cytoreductive surgery plus hyperthermic intraperitoneal chemotherapy versus cytoreductive surgery alone for colorectal peritoneal metastases (PRODIGE 7): a multicentre, randomised, open-label, phase 3 trial. Lancet Oncol. 2021;22(2):256-66.

32. Klaver CEL, Wisselink DD, Punt CJA, et al. Adjuvant hyperthermic intraperitoneal chemotherapy in patients with locally advanced colon cancer (COLOPEC): a multicentre, open-label, randomised trial. Lancet Gastroenterol Hepatol. 2019;4(10):761-70.

33. Abboud K, Andre T, Brunel M, et al. Management of colorectal peritoneal metastases: Expert opinion. $J$ Visc Surg. 2019;156(5):377-9.

34. Ceelen W. HIPEC with oxaliplatin for colorectal peritoneal metastasis: the end of the road? Eur J Surg Oncol. 2019;45(3):400-2.

35. Königsrainer A, Rau B. Cytoreductive surgery (CRS) and hyperthermic intraperitoneal chemotherapy (HIPEC): don't throw the baby out with the bathwater. Pleura Peritoneum. 2018;3(4):20180131.

36. Liberale G, Ameye L, Hendlisz A. PRODIGE 7 should be interpreted with caution. Acta Chir Belg. 2019;119(4):263-6.
37. Yurttas C, Fisher OM, Corts-Guiral D, et al. Cytoreductive surgery and HIPEC in colorectal cancer did we get hold of the wrong end of the stick. Memo Mag Eur Med Oncol. 2020;13:434-9.

38. Brind'Amour A, Webb M, Parapini M, et al. The role of intraperitoneal chemotherapy in the surgical management of pancreatic ductal adenocarcinoma: a systematic review. Clin Exp Metastasis. 2021;38(2):187-96.

39. Helderman RFCPA, Loke DR, Kok HP, et al. Variation in clinical application of hyperthermic intraperitoneal chemotherapy: a review. Cancers (Basel). 2019;11(1):78.

40. Yurttas C, Hoffmann G, Tolios A, et al. Systematic review of variations in hyperthermic intraperitoneal chemotherapy (HIPEC) for peritoneal metastasis from colorectal cancer. J Clin Med. 2018;7(12):567.

41. Löffler MW, Schuster H, Zeck A, et al. Pharmacodynamics of oxaliplatin-derived platinum compounds during hyperthermic intraperitoneal chemotherapy (HIPEC): an emerging aspect supporting the rational design of treatment protocols. Ann Surg Oncol. 2017;24(6):1650-7.

42. Ubink I, Bolhaqueiro ACF, Elias SG, et al. Organoids from colorectal peritoneal metastases as a platform for improving hyperthermic intraperitoneal chemotherapy. $\mathrm{Br} \quad J \quad$ Surg. 2019;106(10):1404-14.

43. Schulz KF, Altman DG, Moher D. CONSORT 2010 statement: updated guidelines for reporting parallel group randomised trials. J Pharmacol Pharmacother. 2010;1(2):100-7.

Publisher's Note Springer Nature remains neutral with regard to jurisdictional claims in published maps and institutional affiliations. 